\title{
Clinical Observation of the Effect of Sevoflurane Inhalation Anesthesia on the Cardiac Function of Elderly Patients Based on Pulsifolex Platform
}

\author{
Zhipeng Liang', Zheng Zuo ${ }^{1}$, Binque Wan ${ }^{1}$, Bingwei $\mathrm{Hu}^{2}$, Ying $\mathrm{Hu}^{2, *}$ \\ ${ }^{1}$ Department of Anesthesiology, Hangzhou Hospital of Traditional Chinese Medicine, Hangzhou, Zhejiang Province, 310007, China. \\ ${ }^{2}$ Department of Anesthesiology, Tongde Hospital of Zhejiang Province, Hangzhou, Zhejiang Province, 310012, China.
}

\begin{abstract}
How to cite this paper: Zhipeng Liang, Zheng Zuo, Binque Wan, Bingwei Hu, Ying Hu. (2022) Clinical Observation of the Effect of Sevoflurane Inhalation Anesthesia on the Cardiac Function of Elderly Patients Based on Pulsifolex Platform. International Journal of Clinical and Experimental Medicine Research, 6(1), 24-30.

DOI: 10.26855/ijcemr.2022.01.005
\end{abstract}

Received: November 6, 2021

Accepted: November 28, 2021

Published: December 9, 2021

*Corresponding author: Ying $\mathrm{Hu}$, Department of Anesthesiology, Tongde Hospital of Zhejiang Province, Hangzhou, Zhejiang Province, 310012, China.

Email: 2693305250@qq.com

\begin{abstract}
Objective: To investigate the effect of sevoflurane inhalation anesthesia on cardiac function of elderly patients based on pulsioflex platform. Methods: from January 2017 to December 2019, 40 elderly patients scheduled for hip replacement were randomly divided into two groups: sevoflurane group (experimental group, $\mathrm{n}=20$ ) and propofol group (control group, $\mathrm{n}=20$ ). Entropy index was used to maintain the depth of anesthesia in all patients. Based on pulsioflex platform, the cardiac work index (CPI), left ventricular contractility index (dpmx), heart rate (HR) and blood pressure (BP) were recorded every 5 minutes before anesthesia, before operation and after operation until the end of operation. The effects of sevoflurane inhalation anesthesia and propofol continuous infusion anesthesia on cardiac function of elderly patients were observed, and the anesthetic efficacy, changes of (CPI, dpmx, HR, BP) and reactions of the two groups were compared. Results: the total effective rate of anesthesia in the observation group was $95.00 \%$, and that in the control group was $90 \%$. The observation group was significantly higher than that in the control group $\left(\chi^{2}=6.05, \mathrm{P}=0.01\right)$. The experimental group was significantly higher than that in the control group, and the difference was statistically significant. Before anesthesia, before operation and at the beginning of operation, the changes of cardiac work index (CPI), left ventricular systolic index (dpmx), heart rate (HR) and blood pressure (BP) in the experimental group were significantly higher than those in the control group, and the difference was statistically significant. The two groups in the hemodynamics faster, hypotension, hypertension, heart rate too fast or too slow, myocardial ischemia signs and other adverse reactions, the experimental group were lower than the control group, the difference was not statistically significant $(P>0.05)$. Conclusion: sevoflurane inhalation anesthesia has definite anesthetic effect on elderly patients undergoing hip replacement. The impact of sevoflurane inhalation anesthesia on cardiac function (CPI, dpmx, HR, BP) of elderly patients monitored by pulsioflex platform is relatively large, and the adverse reactions are relatively small.
\end{abstract}

\section{Keywords}

Pulsioflex Platform, Sevoflurane, Elderly Patients Undergoing Hip Arthroplasty, Impact of Cardiac Function 


\section{Introduction}

Sevoflurane is a new type of inhalation anesthetic, has the characteristics of low blood gas partition coefficient, fast induction, quick recovery, easy adjustment of anesthesia depth, light inhibition of circulation, and less irritation, so it can be used as an ideal inhalation induction drug. In recent years, sevoflurane is also commonly used in adult inhalation induction and anesthesia maintenance, and sevoflurane induction is rapid, good smell, mild inhibition of circulation, no tissue toxicity, mild inhibition of circulation and respiratory function. Sevoflurane has a certain inhibitory effect on circulatory function. It has a direct effect on the myocardium, which is manifested by the decrease of $\mathrm{Ci}$ and $\mathrm{Si}$ and the decrease of blood pressure. The decrease of blood pressure may be the result of sevoflurane's joint action of weakening myocardial contractility, reducing cardiac output and expanding peripheral blood vessels, which is dose-dependent. The effect of inhalation anesthetics on the heart is to prolong the ability of myocardial tolerance to ischemia and reduce the myocardial injury caused by reperfusion by reducing myocardial contractility, myocardial excitability and energy consumption. However, most of these studies (clinical and animal experiments) on sevoflurane are focused on patients undergoing cardiac surgery, and relatively few studies on non cardiac surgery. There are few studies to observe the myocardial inhibition of sevoflurane by continuous monitoring of left ventricular systolic function. In view of the limitations of the above study, the purpose of this study is to explore the effect of sevoflurane on cardiac function of elderly patients by analyzing the changes of CPI and dpmx based on pulsioflex platform during sevoflurane anesth

\section{Materials and methods}

\subsection{General information Research objects}

With the approval of the ethics committee of Hangzhou hospital of traditional Chinese medicine and the consent of the patients, 40 patients with coronary heart disease, aged 65-80 years old, ASA grade II-III and NYHA cardiac function grade II-III, were selected. They were randomly divided into two groups: sevoflurane group (experimental group, $\mathrm{n}=20$ ) and propofol group (control group, $\mathrm{n}=20$ ).

\subsection{ASA grading standard}

Grade I: no organic lesions were found in heart, lung, liver, kidney, brain, endocrine and other important organs; Grade II: mild systemic disease, but in the stage of functional compensation; Grade III: with obvious systemic diseases and early decompensated function; Grade IV: severe systemic disease and decompensated function; Grade V: it is difficult to save the patient's life whether the operation or not.

\subsection{New York Heart Association (NYHA) cardiac function classification}

Grade I: the patient has heart disease, but the physical activity is not limited, daily activities do not cause fatigue, palpitations, dyspnea or angina. Grade II: the physical activity of patients with heart disease is slightly limited, and there are no symptoms at rest. Daily activities can cause fatigue, palpitation, dyspnea or angina pectoris. Grade III: physical activity of patients with heart disease is obviously limited, no symptoms at rest, lighter than daily activities that cause the above symptoms. Grade IV: Patients with heart disease can not engage in any physical activity. There were also symptoms at rest, which worsened after physical activity.

\subsection{Inclusion criteria}

(1) sign informed consent; (2) 65 years old $\leq$ age $\leq 80$ years old; (3) patients undergoing hip replacement under selective general anesthesia; (4) the clinical diagnosis is coronary heart disease; (5) the ejection fraction before operation was more than $40 \%$.

\subsection{Diagnostic basis of coronary heart disease}

(1) there were typical angina pectoris and myocardial infarction, but no severe aortic stenosis, insufficiency, arteritis, coronary embolism or cardiomyopathy. (typical angina pectoris: refers to paroxysmal, located behind the sternum or in the left anterior chest relatively fixed parts of constrictive pain or obvious compression. It can radiate to the left shoulder, left arm, upper abdomen and so on. It often occurs in physical activity, emotional excitement, satiety and cold. I can do it in silence. Rest or nitroglycerin can relieve the symptoms within 3-5 minutes); (2) Male 40, female 45 For patients over the age of 18, ECG showed obvious myocardial ischemia at rest, or ECG exercise test was positive, without other reasons (various heart diseases, autonomic nerve dysfunction, significant anemia, obstructive emphysema, taking digitalis, electrolyte disorder), and two of the following three items: hypertension, 
hypercholesterolemia, diabetes. (during rest, ECG showed obvious myocardial ischemic type, which means that ST segment of ischemic type decreased more than $0.05 \mathrm{MV}$ or T wave inversion more than $2 \mathrm{~mm}$ in the dominant lead of $\mathrm{R}$ wave).

\subsection{Criteria for coronary heart disease patients}

(1) typical angina; (2) the history of myocardial infarction or pathological Q wave in ECG; (3) history of CABG; (4) history of coronary stent implantation.

\subsection{Exclusion criteria}

(1) patients undergoing emergency operation; (2) patients undergoing low-risk surgery, high-risk surgery, cardiac surgery and single leg surgery; (3) patients with ASA grade I, IV and above; (4) patients with congenital heart disease, cardiomyopathy, rheumatic heart disease and pulmonary heart disease; (5) those who cannot cooperate (mental disorder, consciousness disorder, intelligence disorder); (6) HIV positive persons; (7) allergic to the anesthetic used, with a family history of malignant hyperthermia or a history of malignant hyperthermia.

\subsection{Research methods}

\subsubsection{Test drug}

Dexmedetomidine (trade name: eminine), 200 ug/ml, injection (Jiangsu Xinchen Pharmaceutical Co., Ltd.); sufentanil citrate, $50 \mathrm{ug} / \mathrm{ml}$, injection (Hubei Yichang Pharmaceutical Co., Ltd.); vecuronium (trade name: wankesong), $4 \mathrm{mg}$ /bottle, powder (Holland oganon pharmaceutical company); Propofol (trade name: diprivanol), 200 mg/20 ml, injection (Astra) Zeneca Co., Ltd.; sevoflurane (trade name: keteli), $250 \mathrm{ml} /$ bottle, (Jiangsu Xinchen Pharmaceutical Co., Ltd.); hydroxyethyl starch 130/0.4 sodium chloride injection (trade name: wanwen), 500 $\mathrm{ml} / \mathrm{bag}$, (Fresenius cabi Co., Ltd.).

\subsubsection{Main instruments}

Pulsioflex hemodynamic monitoring platform (German pulse medical systems SE); Datex Ohmeda anesthesia machine (Shanghai Lixin Industrial Co., Ltd.); entropy index GE Healthcare Entropy monitoring module (Datex Ohmeda M-Entropy)

\subsection{Anesthesia implementation method}

All 40 patients were randomly divided into sevoflurane anesthesia group (experimental group, $\mathrm{n}=20$ ), propofol anesthesia group (control group, $n=20$ ). Before operation, 12 hours of fasting and 4 hours of drinking were forbidden. The patients were injected with atropine $0.5 \mathrm{mg} 30$ minutes before operation. The patients were injected with lactate Ringer solution and hydroxyethyl starch 130/0.4 sodium chloride injection (WAN Wen), the ratio of crystal gum was 2:1, and the 5-lead ECG was monitored, the open radial artery was connected with pulsioflex system, the heart rate and blood oxygen saturation were monitored by routine anesthesia. The anesthesia depth was maintained by entropy index. The induction of anesthesia was $0.3-0.6 \mathrm{ug} \cdot \mathrm{kg}^{-1}$, sufentanil $0.2-0.4 \mathrm{ug} \cdot \mathrm{kg}^{-1}$, vecuronamine $0.1-0.15 \mathrm{mg} \cdot \mathrm{kg}^{-1}$. The anesthesia maintenance group was continuously inhaled $1-3 \%$ sevoflurane, and the control group was given propofol $4-8 \mathrm{mg} \cdot \mathrm{kg}^{-1} \cdot \mathrm{H}^{-1}$ by intravenous infusion. After the operation, the tracheal catheter was removed according to the routine standard, and the patients were sent to the anesthesia recovery room, and the postoperative analgesia was given.

\subsection{Test indicators}

The patients' heart work index (CPI), left heart systolic force index (dpmx), heart rate (HR), blood pressure (BP) were recorded once every 5 minutes before anesthesia, before operation and after operation, and until the end of operation. For the large hemodynamic changes, first adjust the concentration of sevoflurane inhalation or propofol infusion speed. If the hemodynamic changes are not improved within 1 minute, ephedrine is used for hypotension, nitroglycerin is used for hypertension, esmolol is used for tachycardia, atropine is used for bradycardia. If the ECG of the operation center shows myocardial ischemia, nitroglycerin should be given. Hemodynamic disorders during the anesthesia recovery room were still handled by the researchers.

\subsection{Statistical analysis}

The measurement data are expressed by mean \pm standard deviation, and the counting data are expressed by fre- 
quency. SPSS 17.0 statistical software was used for statistical processing. All the data were measured by chi square test. The mean \pm standard deviation $(-\mathrm{x} \pm \mathrm{s})$ was used for the measurement data. Paired $\mathrm{t}$ test was used for the comparison in the group, and the group t test was used for the comparison between groups. The test level $\alpha=0.05$, the difference was statistically significant with $\mathrm{P}<0.05$.

\section{Results}

1) the total effective rate of anesthesia in the observation group was $95.00 \%$, the total effective rate of anesthesia in the control group was $90 \%$, and the observation group was significantly higher than that of the control group $\left(\chi^{2}\right.$ $=6.05, \mathrm{p}=0.01)$.

Table 1. Comparison of anesthetic efficacy between the two groups $\left(\chi^{2}=6.05, P=0.01\right)$

\begin{tabular}{ccccc}
\hline group & Remarkable effect & Effective & invalid & Total effective rate (\%) \\
\hline Control group $(\mathrm{n}=20)$ & $11(55.00)$ & $7(35.00)$ & $2(10.00)$ & $18(90.00)$ \\
Observation group $(\mathrm{n}=20)$ & $10(50.00)$ & $9(45.00)$ & $1(5.00)$ & $19(95.00)$ \\
$P$ & & - & & 0.01 \\
$\chi^{2}$ & & - & 6.05 \\
\hline
\end{tabular}

2) based on pulsioflex platform, the Cardiac power index (CPI), left ventricular contractility index (dpmx), heart rate (HR) and blood pressure (BP) of the two groups before anesthesia were monitored, as shown in Table 2.

Table 2. Comparison of dpmx, HR, BP and CPI between the two groups $(\bar{x} \pm s)$

\begin{tabular}{ccccc}
\hline group & CPI & dpmx & HR & BP \\
\hline Experimental group $(\mathrm{n}=20)$ & $0.61 \pm 0.18$ & $847 \pm 1.19$ & $80 \pm 2.28$ & $94.31 \pm 1.66$ \\
Control group $(\mathrm{n}=20)$ & $0.63 \pm 0.17$ & $849 \pm 1.41$ & $82.22 \pm 2.43$ & $95.76 \pm 1.09$ \\
$\mathrm{t}$ & 10.112 & 10.274 & 11.214 & 10.278 \\
$P$ & 0.012 & 0.014 & 0.009 & 0.013 \\
\hline
\end{tabular}

3) Based on the pulsioflex platform, the cardiac work index (CPI), left ventricular contractility index (dpmx), heart rate (HR) and blood pressure (BP) of the two groups before operation were monitored, as shown in Table 3.

Table 3. Comparison of dpmx, HR, BP and CPI between the two groups ( $\bar{x} \pm s$ )

\begin{tabular}{ccccc}
\hline group & CPI & dpmx & HR & BP \\
\hline Experimental group $(\mathrm{n}=20)$ & $0.76 \pm 0.25$ & $856 \pm 1.89$ & $90 \pm 3.28$ & $98.31 \pm 1.96$ \\
Control group $(\mathrm{n}=20)$ & $0.79 \pm 0.27$ & $859 \pm 1.61$ & $92.22 \pm 3.43$ & $99.76 \pm 1.49$ \\
$\mathrm{t}$ & 11.162 & 10.974 & 11.814 & 12.678 \\
$P$ & 0.013 & 0.012 & 0.009 & 0.012 \\
\hline
\end{tabular}

4) Cardiac work index (CPI), left ventricular contractility index (dpmx), heart rate (HR) and blood pressure (BP) of the two groups after operation were monitored based on pulsioflex platform, as shown in Table 4.

Table 4. Comparison of dpmx, HR, BP and CPI between the two groups ( $\bar{x} \pm s$ )

\begin{tabular}{ccccc}
\hline group & CPI & dpmx & HR & BP \\
\hline Experimental group $(\mathrm{n}=20)$ & $0.91 \pm 0.12$ & $897 \pm 1.89$ & $98 \pm 1.28$ & $108.31 \pm 1.66$ \\
Control group $(\mathrm{n}=20)$ & $0.93 \pm 0.11$ & $899 \pm 1.49$ & $99 \pm 1.43$ & $109.76 \pm 1.09$ \\
$\mathrm{t}$ & 13.217 & 14.274 & 17.214 & 14.278 \\
$P$ & 0.013 & 0.011 & 0.008 & 0.014 \\
\hline
\end{tabular}


5) The adverse drug reactions of the two groups were shown in Table 5, and the difference was not statistically significant.

Table 5. Comparison of adverse reactions between the two groups (example/\%)

\begin{tabular}{cccccccc}
\hline group & $\begin{array}{c}\text { Rapid } \\
\text { hemodynamics }\end{array}$ & hypotension & hypertension & Tachycardia & bradycardia & $\begin{array}{c}\text { myocardial } \\
\text { ischemia }\end{array}$ & $\begin{array}{c}\text { Hemodynamic } \\
\text { disorder }\end{array}$ \\
\hline $\begin{array}{c}\text { Experimental } \\
\text { group (n=20) }\end{array}$ & 1 & 0 & 2 & 1 & 1 & 0 & 0 \\
$\begin{array}{c}\text { Control group } \\
(\mathrm{n}=20)\end{array}$ & 2 & 1 & 0 & 2 & 0 & 0 & 0 \\
\hline
\end{tabular}

\section{Discussion}

Sevoflurane, a new type of inhalation anesthetic, has the characteristics of low blood gas partition coefficient, fast induction, quick recovery, easy adjustment of anesthesia depth, light inhibition of circulation, and less irritation, so it can be used as an ideal inhalation induction drug. The blood gas partition coefficient of sevoflurane is 0.63 , which is the lowest among inhalation anesthetics except desflurane. In recent years, sevoflurane is also commonly used in adult inhalation induction and anesthesia maintenance, and sevoflurane induction is rapid, good smell, mild inhibition of circulation, no tissue toxicity, mild inhibition of circulation and respiratory function. Sevoflurane has a certain inhibitory effect on circulatory function. It has a direct effect on the myocardium, which is manifested by the decrease of $\mathrm{Ci}$ and $\mathrm{Si}$ and the decrease of blood pressure [1]. The decrease of blood pressure may be the result of sevoflurane's joint action of weakening myocardial contractility, reducing cardiac output and expanding peripheral blood vessels, which is dose-dependent. It has been reported that sevoflurane 1.0 MAC has no obvious effect on tidal volume when inhaled. When anesthesia becomes shallow after operation, there is a reactive increase in respiratory rate, but the blood oxygen saturation remains at a high level, indicating that there is no hypoxia and increase in oxygen consumption.

The effect of inhalation anesthetics on the heart is to prolong the ability of myocardial tolerance to ischemia and reduce the myocardial injury caused by reperfusion by reducing myocardial contractility, myocardial excitability and energy consumption. However, most of these studies (clinical and animal experiments) on sevoflurane are focused on patients undergoing cardiac surgery, and relatively few studies on non cardiac surgery.

Propofol is a rapid and short acting intravenous general anesthetic, which has the characteristics of strong anesthetic efficacy, fast onset, short duration, rapid and stable recovery, and few adverse reactions. However, propofol has a strong respiratory and circulatory inhibition effect, and has the effect of reducing sympathetic nerve activity. The circulatory inhibition effect of propofol is positively correlated with injection speed and dose. Therefore, sevoflurane and propofol represent the development trend of inhalation anesthesia and intravenous anesthesia respectively. People pay more attention to them and their clinical application is becoming more and more extensive. For a long time, the clinical diagnosis of acute myocardial infarction depends on the abnormal increase of serum myocardial enzymes, especially creatine kinase MB (CK-MB) concentration. In recent years, studies have shown that the detection of cardiac markers, especially cardiac troponin I (cTnI), plays an important role in the diagnosis of acute myocardial infarction. It has become one of the most specific and sensitive serum markers for monitoring acute myocardial infarction [2], and has been widely used in clinical diagnosis. Cardiac troponin and myocardial enzymes appear earlier in the blood when myocardial damage occurs, which has high sensitivity and specificity for the diagnosis of perioperative myocardial damage, and can be used as myocardial protection markers to evaluate the effect of perioperative myocardial protection and judge the prognosis of patients [3, 4].

Left ventricular systolic function is an important index of clinical circulatory function, especially in patients with acute and severe perioperative period. Early evaluation of blood volume, vascular tension and cardiac function according to hemodynamics, correction of cardiac insufficiency and poor tissue perfusion have extremely important clinical value for improving the prognosis of patients. Left ventricular systolic function is the most important part of cardiac function. The proAQT minimally invasive hemodynamic monitoring technology (arterial pulse profile analysis technology) is a minimally invasive method, which can monitor the cardiac work index (CPI) and left ventricular contractility index (dpmx). Dpmx is the largest slope of the arterial pressure curve ( $\triangle$ Pmax/ $\Delta T)$, which can be used to evaluate the systolic force of the left heart. CPI, calculated by the combination of pressure (map) and flow (CO), reflects the output capacity of left ventricular output and is the best index to predict the in-hospital mor- 
tality of cardiogenic shock. Some studies have shown that pulsioflex platform can track the change of Ci in critically ill patients with hemodynamic instability [5]. At the same time, some studies have shown that pulsioflex platform technology used for monitoring patients with abdominal surgery can reduce the incidence of postoperative complications and hospital stay [6].

With the development of human society, the average life expectancy of the population in various countries continues to extend, and the sharp increase of the elderly population will bring a series of social problems. Therefore, the relevant research on the field of elderly will play an extremely important role in the construction of health services in China. The incidence rate of coronary heart disease has been increasing year by year. With the development of aging population, the cardiac adverse events occurring in elderly patients with coronary heart disease undergoing non cardiac surgery are attracting more and more attention. Coronary heart disease (CHD) is a series of serious myocardial ischemia diseases, such as angina pectoris, myocardial infarction and so on. The incidence rate and mortality rate of coronary heart disease in the developed countries in Europe and the United States ranks the top three. The incidence rate of coronary heart disease is $2 \%$ to $4 \%$ in China over 40 years old, and the trend is increasing year by year [7]. Myocardial ischemia usually indicates the decrease of coronary circulation reserve capacity, or when various factors lead to the increase of cardiac load, coronary blood flow cannot increase with the increase of myocardial oxygen demand. The incidence of myocardial ischemia in patients with coronary heart disease during noncardiac surgery is as high as 38\%-67\% [8]. Studies have shown that elderly patients with coronary heart disease in the emergency and large and medium-sized surgery, it is very easy to induce and aggravate a variety of cardiac events [9]. Perioperative cardiac events are the main cause of perioperative death. Postoperative myocardial ischemia is an important predictor of perioperative cardiac events. Therefore, understanding the characteristics and regularity of perioperative myocardial ischemia can take preventive measures in time, which is helpful to reduce the perioperative mortality and improve the prognosis of patients.

According to the current statistics, about $28 \%$ of the patients who plan to undergo elective noncardiac surgery have coronary heart disease or multiple cardiac risk factors. These patients are prone to cardiovascular and cerebrovascular accidents during perioperative period, so maintaining the stability of cardiovascular function of these patients has important clinical significance for reducing postoperative complications, improving prognosis, reducing mortality and disability rate. In addition to the patient's preoperative condition, surgical stimulation and other factors, the influence of different anesthesia methods and drugs on perioperative cardiac function cannot be ignored. Studies have shown that halogenated volatile anesthetics such as sevoflurane, enflurane and isoflurane can dilate coronary artery, reduce myocardial infarction area and enhance mechanical function after myocardial ischemia, so as to play the role of myocardial protection. The mechanism of action is as follows: (1) activating mitochondrial KATP channel to produce "ischemic preconditioning" effect; (2) reducing neutrophil aggregation and platelet adhesion after myocardial ischemia; (3) increasing collateral blood circulation in ischemic area; (4) reducing ATP consumption. Similarly, opioids such as morphine, fentanyl and sufentanil can also reduce myocardial ischemia-reperfusion injury by activating delta receptor, reducing the expression of cell adhesion factor and inhibiting neutrophil translocation. Intravenous anesthetics such as propofol can improve the mechanical function and reduce the size of myocardial infarction by reducing the production of free radicals, reducing calcium influx and inhibiting the activity of neutrophils.

\section{Conclusion}

The results of this study showed that the total effective rate of anesthesia in the observation group was 95.00\%, and that in the control group was $90 \%$. The total effective rate of anesthesia in the observation group was significantly higher than that in the control group $\left(\chi^{2}=6.05, \mathrm{P}=0.01\right)$, the difference was statistically significant. The changes of cardiac work index (CPI), left ventricular contractility index (dpmx), heart rate (HR) and blood pressure (BP) of the two groups were monitored based on pulsioflex platform, which was statistically significant. Adverse reactions were not statistically significant.

\section{Acknowledgements}

Fund Project: Hangzhou Medical and health science and technology plan project (2018a61); Zhejiang Medical Association project (2017zyc-a34). 


\section{References}

[1] Zhang Youhua, Li Xiebing. (2016). The value of cTnI, CK-MB in the diagnosis of acute myocardial infarction [J]. Fujian Journal of Medicine, 28(3): 48-53.

[2] Ye Ping'an, Liang Qi. (2013). The value of cardiac troponin I in the diagnosis of perioperative myocardial injury [J]. Chinese Journal of Anesthesiology, 15(2): 141-142.

[3] Riou, B. (2014). Troponin: important in severe trauma and a first step in the biological marker revolution [J]. Anesthesiology, 101: $1259-1260$.

[4] Penttila, I., Penttila, K., Rantanen, T. (2016). Laboratory diagnosis of patients with acute chest pain [J]. Clin Chem Lab Med., 38: 187-97.

[5] Vijver, K. V. D., Pigozzi, C., Vervliet, L., et al. (2014). Validation of less-invasive hemodynamic monitoring with Pulsioflex in critically ill patients: interim results of a multicentre study [J]. Critical Care, 17(S2): 193-200.

[6] Salzwedel, C., Puig, J., Carstens, A., et al. (2015). Perioperative goal-directed hemodynamic therapy based on radial arterial pulse pressure variation and continuous cardiac index trending reduces postoperative complications after major abdominal surgery: a multi-center, prospective, randomized study [J]. Critical Care, 17(5): 192-198.

[7] Lee, T. H., Marcantonio, E. R., Mangione, C. M., et al. (2016). Derivation and prospective validation of a simple index for prediction of cardiac risk of major non-cardiac surgery [J]. Circulation, 100(10): 1043-1049.

[8] Butterworth, J., Furgerg, C. D. (2014). Improving cardiac outcomes after non-cardiac surgery [J]. Anesth Analg., 97(3): 613-615.

[9] Devereaux, P. J., Goldman, L., Yusuf, S., et al. (2018). Surveillance and prevention of major perioperative ischemic cardiac events in patients undergoing non-cardiac surgery: a review [J]. Can Med Assoc., 173(7): 779-7881.

[10] Kersten, J. R., Sehmeling, T., Tessmer, J., et al. (2019). Sevoflurane selectively increases coronary collateral blood flow independent of K-ATP channels in vivo. Anesthesiology, 90: 246-256.

[11] Conzen, P. F., Fischer, S., Detter, C., Peter, K. (2018). Sevoflurane provides greater protection of the myocardium than propofol in patients undergoing off-pump coronary artery bypass surgery [J]. Anesthesiology, 99: 826-833.

[12] Bein, B., Renner, J., Caliebe, D., et al. (2018). Sevoflurane but not propofol preserves myocardial function during minimally invasive direct coronary artery bypass surgery [J]. Anesth Analg., 100: 610-616. 